\title{
A Parallel Pressure Projection Stabilized Finite Element Method for Stokes Equation with Nonlinear Slip Boundary Conditions
}

\author{
Kangrui Zhou and Yueqiang Shang* \\ School of Mathematics and Statistics, Southwest University, Chongqing, 400715, China
}

Received 25 June 2019; Accepted (in revised version) 18 October 2019

\begin{abstract}
For the low-order finite element pair $P_{1}-P_{1}$, based on full domain partition technique, a parallel pressure projection stabilized finite element algorithm for the Stokes equation with nonlinear slip boundary conditions is designed and analyzed. From the definition of the subdifferential, the variational formulation of this equation is the variational inequality problem of the second kind. Each subproblem is a global problem on the composite grid, which is easy to program and implement. The optimal error estimates of the approximate solutions are obtained by theoretical analysis since the appropriate stabilization parameter is chosen. Finally, some numerical results are given to demonstrate the hight efficiency of the parallel stabilized finite element algorithm.
\end{abstract}

AMS subject classifications: 68W10, 76M10, 76D07

Key words: Stokes equations, nonlinear slip boundary conditions, pressure projection, full domain partition, parallel stabilized finite element algorithm.

\section{Introduction}

In this paper, we shall study the incompressible Stokes problem:

$$
\begin{cases}-v \Delta u+\nabla p=f & \text { in } \Omega, \\ \nabla \cdot u=0 & \text { in } \Omega,\end{cases}
$$

with the following homogeneous Dirichlet boundary condition on $\Gamma$ and nonlinear slip boundary conditions on $S$ :

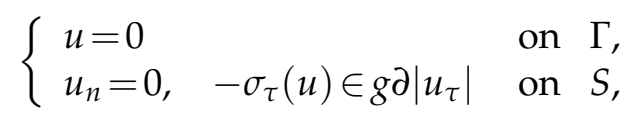

\footnotetext{
*Corresponding author.

Emails: zhoukangrui@126.com (K. R. Zhou),yqshang@swu.edu.cn (Y. Q. Shang)
} 
where $\Omega \subset \mathbb{R}^{2}$ is a bounded convex domain with a Lipschitz-continuous boundary $\partial \Omega$. $\Gamma \cap S=\varnothing, \overline{\Gamma \cup S}=\partial \Omega . g \geq 0$ is a scalar function stands for the barrier or threshold slip function; $u_{n}=u \cdot n$ and $u_{\tau}=u \cdot \tau$ are the normal and tangential components of the velocity on $S$ respectively; the Cauchy stress vector $\sigma$ is defined as $\sigma_{i}=\sigma_{i}(u, p)=\left(v e_{i j}(u)-p \delta_{i j}\right) n_{j}$, here $e_{i j}(u)=\frac{\partial u_{i}}{\partial x^{j}}+\frac{\partial u_{j}}{\partial x^{i}}, \delta_{i j}=1$ if $i=j$ and $\delta_{i j}=0$ if $i \neq j$, write $\sigma_{\tau}(u)=\sigma-\sigma_{n} n$ is the tangential component of $\sigma$. Set $\phi: \mathbb{R} \rightarrow \overline{\mathbb{R}}=(-\infty,+\infty]$ and it contains the properties of convexity and weak semi-continuity from below ( $\phi$ is not identical with $+\infty$ ). The subdifferential set $\partial \phi(a)$ denotes a subdifferential of the function $\phi$ at the point $a$ is defined by: $\partial \phi(a)=\{b \in$ $\mathbb{R}: \phi(h)-\phi(a) \geq b(h-a), \forall h \in \mathbb{R}\}$.

As a fundamental equations of fluid mechanics, Stokes equations are widely used, and relevant mathematical research can be found in [1,2]. In some fluid problems, such as blood flow in a vein of an arterial sclerosis patient, the avalanches in water and rocks, flow through a canal or a drainpipe with its bottom covered by sherbet of mud and pebbles, oil flows above or below the sand, the traditional Dirichlet boundary conditions are no longer applicable, so there is practical application value in studying Stokes equations with nonlinear slip boundary conditions. The boundary condition (1.2) was introduced by Fujita in [3], subsequently, there have been many studies about the properties of the solution (cf. [3-9]), including existence, uniqueness, regularity, and continuous dependence on data for the Stokes and Navier-Stokes equations with boundary conditions (1.2). Also, there are a lot of finite element methods for Stokes and Navier-Stokes equations which satisfying inf-sup condition, like the penalty finite element methods in [10-12], discontinuous Galerkin methods in [13] and so on.

The low-order finite element pair $P_{1}-P_{1}$ does not satisfy the inf-sup condition, however, it has been widely used in practical calculations due to its simple and regular data structure, which makes calculations small and accurate. In order to solve the pressure oscillation caused by low-order finite element pair $P_{1}-P_{1}$, many stabilization techniques are used to compensate for the inf-sup condition, for example, two-level penalized finite element methods [14], regularization method [15], local Gauss integrations method [16], variational multiscale method [17] and pressure projection stabilization method [18]. The pressure projection stabilization method proposed in this paper is based on the literature [19]. Compared with other stabilization methods, this method does not need to calculate high-order derivatives or boundary integrals, and there is no grid nesting.

On the basis of literature [20,21], we propose a more efficient algorithm for equations (1.1)-(1.2), which is the parallel algorithm. Then, by choosing the appropriate stabilization parameters, we can obtain the optimal error estimates for approximate solutions. The idea of local and parallel finite element computations was from Xu's work [22], which have become a hot research topic with the advent of parallel computers. However, there is little research on the local and parallel computations for the Stokes or NavierStokes equations with nonlinear slip boundary conditions. A finite element solution of some partial differential equations usually consists of low-frequency components and high frequency components. The low-frequency components describe a wide range of 
information, where the high-frequency components describe specific details. The global behavior of a finite element solution is mostly governed by low frequencies and the local behavior is mostly governed by high frequencies. The main idea of this method is to use a composite grid to solve a global problem to obtain a local approximate solution in a given subdomain, which contains the most of the degree of freedom in the composite grid it represents for. The composite grid is constituted by fine elements in the given subdomain and coarse elements on the rest of domain (see Fig. 1).

The paper's frame is as follows: in Section 2, we introduce some preliminary materials, assumptions of mixed finite element spaces, pressure projection stabilization method and its error estimates also be given in this section. In Section 3, the local stabilized finite element method is given and error estimates are derived. In Section 4, based on local stabilized finite element method in Section 3, we introduce and analyze the parallel stabilized finite element algorithms where the full domain partition technique is applied. In Section 5, numerical results which support our theory are presented. Finally, conclusions in Section 6.

\section{Stokes equation with nonlinear slip boundary condition}

\subsection{Preliminaries}

In Hilbert space [23], $\|\cdot\|_{k}$ stands for the norm of $H^{k}(\Omega)^{2},(\cdot, \cdot)$ and $\|\cdot\|$ stand for the inner product and the norm of $L^{2}(\Omega)^{2}$. Some spaces used in this paper are defined by:

$$
\begin{aligned}
& V=\left\{u \in H_{1}(\Omega)^{2},\left.u\right|_{\Gamma}=0,\left.u \cdot n\right|_{S}=0\right\}, \quad V_{0}=H_{0}^{1}(\Omega)^{2}, \\
& M=L_{0}^{2}(\Omega)=\left\{q \in L^{2}(\Omega), \int_{\Omega} q d x=0\right\} .
\end{aligned}
$$

Also the inner product and the norm in $V$ can be written as $(\nabla \cdot, \nabla \cdot)$ and $\|\cdot\|_{V}=\|\nabla \cdot\|$, respectively. The space $H^{-1}(\Omega)^{2}$ will also be used which is the dual of $H^{1}(\Omega)^{2}$. For $D \subset G \subset \Omega$, the notation $D \subset \subset G$ stands for that dist $(\partial D / \partial \Omega, \partial G / \partial \Omega)>0$. Throughout this paper we shall use the letter $c$ (with or without subscripts) to denote a generic positive constant which may stand for different values at its different occurrences. We define $a(\cdot, \cdot), b(\cdot, \cdot)$ as

$$
a(u, v)=v(\nabla u, \nabla v), \quad d(v, q)=(\nabla \cdot v, q) \quad \forall u, v \in V, \quad q \in M .
$$

With the definition of above notation and subdifferential set, the formulation of (1.1)-(1.2) read as follows:

Find $(u, p) \in V \times M$ such that

$$
\begin{cases}a(u, v-u)+j\left(v_{\tau}\right)-j\left(u_{\tau}\right)-d(v-u, p) \geq(f, v-u), & \forall v \in V, \\ d(u, q)=0, & \forall q \in M,\end{cases}
$$


where

$$
j(\zeta)=\int_{S} g|\zeta| d s, \quad \zeta \in H^{\frac{1}{2}}(S) .
$$

We define bilinear form $B:(V, M) \times(V, M) \rightarrow \mathbb{R}$ as follows:

$$
B(u, p ; v, q)=a(u, v)-d(v, p)+d(u, q) .
$$

The following existence and uniqueness theorem of the solution to the problem (2.1) are classical and we can see Fujita [3] and Saito [9] for details.

Theorem 2.1. Assume $f \in L^{2}(\Omega)^{2}$ and $g \in L^{2}(S)$. Then the variational inequality problem (2.1) admits a unique solution $(u, p) \in V \times M$ such that

$$
\|u\|_{V}+\|p\| \leq c\left(\|f\|+\|g\|_{L^{2}(S)}\right),
$$

where $c>0$ depends only on $v$ and $\Omega$. Furthermore, if $\partial \Omega$ is sufficiently smooth, then the weak solution is also regular solution, ie., $u \in H^{2}(\Omega)^{2}$ and $p \in H^{1}(\Omega)$, such that

$$
\|u\|_{2}+\|p\|_{1} \leq c\left(\|f\|+\|g\|_{L^{2}(S)}\right),
$$

where $c>0$ depends only on $v$ and $\Omega$.

\subsection{Mixed finite element space}

Assume $T^{\mu}(\Omega)=\{K\}(\mu=h, H)$ to be regular triangle meshes of $\Omega$ with mesh size $\mu(x)$, whose value is the diameter $\mu_{K}$ of the element $K$ containing $x$. Basic assumption on the mesh is that it is not exceedingly over-refined locally:

A0. There exists $\gamma \geq 1$ such that

$$
\mu_{\Omega}^{\gamma} \leq c \mu(x), \quad \forall x \in \Omega,
$$

where $\mu_{\Omega}=\max _{x \in \Omega} \mu(x)$ is the largest mesh size of $T^{\mu}(\Omega)$. We defines the velocity finite element space $V_{\mu}$ and the pressure finite element space $M_{\mu}$ as follows:

$$
\begin{aligned}
& V_{\mu}=\left\{v \in V:\left.v\right|_{K} \in P_{1}(K)^{2}, \forall K \in T^{\mu}(\Omega)\right\}, \\
& M_{\mu}=\left\{q \in M:\left.q\right|_{K} \in P_{1}(K), \forall K \in T^{\mu}(\Omega)\right\},
\end{aligned}
$$

where $P_{1}$ is the space of complete linear polynomials. Associated with the mesh $T^{h}(\Omega)$, let $V_{h} \subset V, M_{h} \subset M$ be two finite element subspaces on $\Omega$. Given $G \subset \subset \Omega$, we define $V_{h}(G)$, $M_{h}(G)$ and $T^{h}(G)$ to be the restriction of $V_{h}, M_{h}$ and $T^{h}(\Omega)$ to $G$, and

$$
V_{0}^{h}(G)=\left\{v \in V_{h}(\Omega): \operatorname{supp} v \subset \subset G\right\}, \quad M_{0}^{h}(G)=\left\{q \in M_{h}(\Omega): \operatorname{supp} q \subset \subset G\right\} .
$$

Next, we shall give our basic assumptions on the mixed finite element spaces (cf. $[24,25])$. 
A1 Approximation. For each $(u, p) \in H^{t+1}(G)^{2} \times H^{t}(G)(t \geq 1)$, there exists $(v, q) \in V_{h}(G) \times$ $M_{h}(G)$ such that

$$
\begin{gathered}
\inf _{v \in V_{0}^{h}(\Omega)}\left(\left\|h^{-1}(u-v)\right\|_{0, G}+\|u-v\|_{1, G}\right) \leq c h\|u\|_{2, G}, \\
\inf _{q \in M_{0}^{h}(\Omega)}\left(\left\|h^{-1}(p-q)\right\|_{-1, G}+\|p-q\|_{0, G}\right) \leq c h\|p\|_{1, G} .
\end{gathered}
$$

A2 Inverse estimate. For any $(v, q) \in V_{h}(G) \times M_{h}(G)$, it is hold that

$$
\|v\|_{1, \Omega} \leq c\left\|h^{-1} v\right\|_{0, G}, \quad\|q\|_{0, G} \leq c\left\|h^{-1} q\right\|_{-1, G} .
$$

A3 Superapproximation. For $G \subset \Omega_{0}$, let $\omega \in C_{0}^{\infty}(\Omega)$ with supp $\omega \subset \subset G$. And for any $(u, p) \in V_{h}(G) \times M_{h}(G)$, there exist $(v, q) \in V_{0}^{h}(G) \times M_{0}^{h}(G)$ such that

$$
\left\|h^{-1}(\omega u-v)\right\|_{1, G} \leq c\|u\|_{1, G}, \quad\left\|h^{-1}(\omega p-q)\right\|_{0, G} \leq c\|p\|_{0, G} .
$$

\subsection{Pressure projection stabilized finite element method}

Next, we will pose the pressure projection stabilized finite element approximation formulations of Stokes equations with nonlinear slip boundary conditions. Since the low-order finite element pair does not satisfy the inf-sup condition, to compensate for the deficiency, the bilinear form of the stabilization term is defined as follows:

$$
G(p, q)=\alpha(p-\Pi p, q-\Pi q),
$$

where $\Pi: M \rightarrow P_{0}$ is local pressure projection, $\alpha,(0<\alpha<1)$ is stabilization parameter, which satisfies $\alpha=\mathcal{O}(h)$ in this paper. Based on the above assumptions, refer [19], the pressure projection stabilized finite element approximation formulations of (2.1) is:

Find $\left(u_{h}, p_{h}\right) \in\left(V_{h}, M_{h}\right)$ such that

$$
\begin{cases}a\left(u_{h}, v_{h}-u_{h}\right)+j\left(v_{h \tau}\right)-j\left(u_{h \tau}\right)-d\left(v_{h}-u_{h}, p_{h}\right) \geq\left(f, v_{h}-u_{h}\right), & \forall v_{h} \in V_{h}, \\ d\left(u_{h}, q_{h}\right)+G\left(p_{h}, q_{h}\right)=0, & \forall q_{h} \in M_{h} .\end{cases}
$$

The bilinear form $B_{h}$ is defined as

$$
B_{h}\left(u_{h}, p_{h} ; v_{h}, q_{h}\right)=a\left(u_{h}, v_{h}\right)-d\left(v_{h}, p_{h}\right)+d\left(u_{h}, q_{h}\right)+G\left(p_{h}, q_{h}\right), \quad \forall\left(v_{h}, q_{h}\right) \in\left(V_{h}, M_{h}\right) .
$$

Regarding the bilinear term $B_{h}$, the stability theorem established in the local pressure projection stabilization finite element method is given below (cf. [19]).

Theorem 2.2. For $\forall p \in M$, assume $\Pi$ is continuous as an operator $\Pi: M \rightarrow P_{0}$ :

$$
\|\Pi p\| \leq c\|p\|, \quad q \in M, \quad\|p-\Pi p\| \leq c h\|p\|_{1}, \quad q \in H^{1}(\Omega),
$$


then there is a constant $\beta \geq 0$ independent of $h$, satisfying

$$
\begin{array}{ll}
\left|B_{h}\left(u_{h}, p_{h} ; v_{h}, q_{h}\right)\right| \leq c\left(\left\|u_{h}\right\|_{V}+\left\|p_{h}\right\|\right)\left(\left\|v_{h}\right\|_{V}+\|q\|\right), & \forall\left(u_{h}, p_{h}\right),\left(v_{h}, q_{h}\right) \in\left(V_{h}, M_{h}\right), \\
\beta\left(\left\|u_{h}\right\|_{V}+\left\|p_{h}\right\|\right) \leq \sup _{\left(v_{h}, q_{h}\right) \in\left(\widetilde{V}_{h}, M_{h}\right)} \frac{B_{h}\left(u_{h}, p_{h} ; v_{h}, q_{h}\right)}{\left\|v_{h}\right\|_{V}+\|q\|}, & \forall\left(u_{h}, p_{h}\right) \in\left(\widetilde{V}_{h}, M_{h}\right),
\end{array}
$$

where $\widetilde{V}_{h}=\left\{v \in V_{0}:\left.v\right|_{K} \in P_{1}(K)^{2}, \forall K \in T^{h}\right\}$ be the finite element subspace of $V_{0}$.

The steady homogeneous and inhomogeneous Stokes system with linear slip boundary conditions without subdifferential property have been studied form the theoretical view point by Beirão da Veiga [26-28]. To estimate low-order errors $\left\|u-u_{h}\right\|+\left\|p-p_{h}\right\|_{-1}$, we consider the following Stokes problems with linear slip boundary condition.

$$
\begin{cases}-v \Delta w-\nabla \pi=\varphi & \text { in } \Omega, \\ -\nabla \cdot w=\psi & \text { in } \Omega, \\ w=0 & \text { on } \Gamma, \\ w_{n}=0, \quad \sigma_{\tau}(w)=0 & \text { on } S,\end{cases}
$$

which admits a unique solution $(w, \pi) \in H^{2}(\Omega)^{2} \cap V \times H^{1}(\Omega) \cap M$ such that

$$
\|w\|_{2}+\|\pi\|_{1} \leq c\left(\|\varphi\|+\|\psi\|_{1}\right)
$$

where $c>0$ independent of $h$. Let $w_{h} \in \widetilde{V}_{h} \subset V_{0}$ and $\pi_{h} \in M_{h}$ be the stabilized finite element approximation solution of (2.7) and satisfy

$$
\begin{cases}a\left(w_{h}, v_{h}\right)+d\left(w_{h}, \pi_{h}\right)=\left(\varphi, v_{h}\right), & \forall v_{h} \in V_{h} \\ -d\left(w_{h}, q_{h}\right)+G\left(\pi_{h}, q_{h}\right)=\left(\psi, q_{h}\right), & \forall q_{h} \in M_{h}\end{cases}
$$

then it is well known that

$$
\left\|w-w_{h}\right\|_{V}+\left\|\pi-\pi_{h}\right\| \leq \operatorname{ch}\left(\|\varphi\|+\|\psi\|_{1}\right),
$$

where $c>0$ independent of $h$.

Theorem 2.3. Let $\left(u_{h}, p_{h}\right)$ be the finite element approximation solution of (2.5), then the following inequalities hold

$$
\begin{aligned}
& \left\|u-u_{h}\right\|_{V}+\left\|p-p_{h}\right\| \leq \operatorname{ch}\left(\|f\|+\|g\|_{L^{2}(S)}\right), \\
& \left\|u-u_{h}\right\|+\left\|p-p_{h}\right\|_{-1} \leq \operatorname{ch}^{2}\left(\|f\|+\|g\|_{L^{2}(S)}\right),
\end{aligned}
$$

where $c>0$ independent of $h$. 
Proof. The estimate $\left\|u-u_{h}\right\|_{V}+\left\|p-p_{h}\right\|$ is similar to [20]. Now, we give the error estimate $\left\|u-u_{h}\right\|+\left\|p-p_{h}\right\|_{-1}$ by the Aubin-Nitsche's technique. Setting $v=u \pm w_{h}$ in (2.1) yields

$$
a\left(u, w_{h}\right)-d\left(w_{h}, p\right)=\left(f, w_{h}\right), \quad \forall w_{h} \in \widetilde{V}_{h} .
$$

Setting $v_{h}=u_{h} \pm w_{h}$ in (2.5) yields

$$
a\left(u_{h}, w_{h}\right)-d\left(w_{h}, p_{h}\right)=\left(f, w_{h}\right), \quad \forall w_{h} \in \widetilde{V}_{h} .
$$

And hence

$$
a\left(u-u_{h}, w_{h}\right)=d\left(w_{h}, p-p_{h}\right), \quad \forall w_{h} \in \widetilde{V}_{h} .
$$

Taking $v=u-u_{h}, q=p-p_{h}$ in (2.9), we have

$$
\begin{aligned}
& \left(\varphi, u-u_{h}\right)+\left(\psi, p-p_{h}\right) \\
= & a\left(w, u-u_{h}\right)+d\left(u-u_{h}, \pi\right)-d\left(w, p-p_{h}\right) \\
= & a\left(w-w_{h}, u-u_{h}\right)+a\left(w_{h}, u-u_{h}\right)+d\left(u-u_{h}, \pi-\pi_{h}\right) \\
& \quad+d\left(u-u_{h}, \pi_{h}\right)-d\left(w-w_{h}, p-p_{h}\right)-d\left(w_{h}, p-p_{h}\right),
\end{aligned}
$$

according to (2.12) and $d\left(u-u_{h}, \pi_{h}\right)=G\left(p_{h}, \pi_{h}\right),(2.13)$ is equal to

$$
\begin{aligned}
& \left(\varphi, u-u_{h}\right)+\left(\psi, p-p_{h}\right) \\
= & a\left(w-w_{h}, u-u_{h}\right)+d\left(u-u_{h}, \pi-\pi_{h}\right) \\
& -d\left(w-w_{h}, p-p_{h}\right)+G\left(p_{h}, \pi_{h}\right) .
\end{aligned}
$$

Apply (2.6), Assumption A2, (2.8) and (2.10), we get

$$
\begin{aligned}
& G\left(p_{h}, \pi_{h}\right)=\alpha\left(p_{h}-\Pi p_{h}, \pi_{h}-\Pi \pi_{h}\right) \leq \alpha\left\|p_{h}-\Pi p_{h}\right\| \cdot\left\|\pi_{h}-\Pi \pi_{h}\right\| \\
\leq & \alpha\left(\left\|p-p_{h}\right\|+\|p-\Pi p\|+\left\|\Pi p-\Pi p_{h}\right\|\right)\left(\left\|\pi-\pi_{h}\right\|+\|\pi-\Pi \pi\|+\| \Pi \pi-\Pi \pi_{h} \mid\right) \\
\leq & c \alpha\left(\left\|p-p_{h}\right\|+h\|p\|_{1}\right)\left(\left\|\pi-\pi_{h}\right\|+h\|\pi\|_{1}\right) \\
\leq & c \alpha h\left\|p-p_{h}\right\|\left(\|\varphi\|+\|\psi\|_{1}\right)+c \alpha h^{2}\|p\|_{1}\left(\|\varphi\|+\|\psi\|_{1}\right) .
\end{aligned}
$$

Substituting the above inequalities into (2.14) yields

$$
\begin{aligned}
& \quad\left(\varphi, u-u_{h}\right)+\left(\psi, p-p_{h}\right) \\
& \leq c(1+\alpha) h\left(\left\|u-u_{h}\right\|_{V}+\left\|p-p_{h}\right\|\right)\left(\|\varphi\|+\|\psi\|_{1}\right) \\
& \quad+c \alpha h^{2}\|p\|_{1}\left(\|\varphi\|+\|\psi\|_{1}\right) .
\end{aligned}
$$

Apply Theorem 2.1, we have

$$
\begin{aligned}
& \left\|u-u_{h}\right\|+\left\|p-p_{h}\right\|_{-1} \\
\leq & c(1+\alpha) h\left(\left\|u-u_{h}\right\|_{V}+\left\|p-p_{h}\right\|\right)+c \alpha h^{2}\|p\|_{1} \\
\leq & c h^{2}\left(\|f\|+\|g\|_{L^{2}(S)}\right) .
\end{aligned}
$$

We complete the proof. 


\section{Local stabilized finite element algorithms}

In this section, the local pressure projection stabilized finite element algorithm for Stokes equations with nonlinear slip boundary condition will be presented and analyzed, priori error estimates will also be derived.

Assume $T^{H}(\Omega)$ to be a global coarse grid with the mesh size $H \gg h$ and $T^{h}\left(\Omega_{0}\right)$ is a local fine grid with mesh size $h$ where $\Omega_{0}$ is obtained by enlarging subdomain $D$ slightly (i.e. $D \subset \subset \Omega_{0} \subset \Omega$ ). The global grid $T^{H, h}(\Omega)$ is a composite mesh which is fine around the subdomain $D$ with size $h$ in such a way that $T^{H, h}\left(\Omega_{0}\right)=T^{h}\left(\Omega_{0}\right)$ and coarse on the rest area with size $H \gg h$. We can refining the coarse grid $T^{H}(\Omega)$ locally to acquire this composite mesh $T^{H, h}(\Omega)$ and use some adaptive processes to make it compatibility (it is compatible for a mesh or triangulation means the intersection of two elements is either empty, a common vertex, a common side or a common face). For mesh $T^{H, h}(\Omega)$, the corresponding finite element space is denote as $V_{H, h}(\Omega) \subset V, M_{H, h}(\Omega) \subset M$ which satisfy the Assumptions A1-A3.

\section{Algorithm 3.1. Local stabilized finite element algorithms.}

Find $\left(u_{H}^{h}, p_{H}^{h}\right) \in V_{H, h}(\Omega) \times M_{H, h}(\Omega)$ such that

$$
\begin{cases}a\left(u_{H}^{h}, v-u_{H}^{h}\right)+j\left(v_{\tau}\right)-j\left(u_{H \tau}^{h}\right)-d\left(v-u_{H}^{h}, p_{H}^{h}\right) \geq\left(f, v-u_{H}^{h}\right), & \forall v \in V_{H, h}(\Omega), \\ \left.d\left(u_{H}^{h}, q\right)+G\left(p_{H}^{h}, q\right)=0\right), & \forall q \in M_{H, h}(\Omega) .\end{cases}
$$

To analyze the above mentioned local algorithm, the following Lemma 3.2 is vital. The proof is similar to the Lemma 3.2 in the $[29,30]$.

Lemma 3.1. Let $\omega \in C_{0}^{\infty}(\Omega)$ such that supp $\omega \subset \subset \Omega_{0}$. Then

$$
\|\omega w\|_{1, \Omega}^{2} \leq c a\left(w, \omega^{2} w\right)+c\|w\|_{0, \Omega_{0}}^{2}, \quad \forall w \in H_{0}^{1}(\Omega)^{2} .
$$

Lemma 3.2. Assume $g \in H^{-1}(\Omega)^{2}, D \subset \subset \Omega_{0} \subset \Omega$, Assumptions A1-A3 hold and the stabilization parameter is taken as $\alpha=\mathcal{O}(h)$. Then $(w, r) \in V_{h} \times M_{h}$ satisfying

$$
\begin{cases}a(w, v)-d(v, r)=(g, v), & \forall v \in V_{h}\left(\Omega_{0}\right), \\ d(w, q)+G(r, q)=0, & \forall q \in M_{h}\left(\Omega_{0}\right),\end{cases}
$$

has the following local estimate

$$
\|w\|_{1, D}+\|r\|_{0, D} \leq c\left(\|w\|_{0, \Omega_{0}}+\|r\|_{-1, \Omega_{0}}+\|g\|_{-1, \Omega_{0}}\right) .
$$

Proof. Let $s$ be an integer such that $s \geq \max \{2 \gamma-1, \gamma+1\}$. $D_{j}$ and $\Omega_{j}(j=1, \cdots, s)$ satisfy

$$
\begin{aligned}
& D_{1} \subset \subset D_{2} \subset \subset \cdots \subset \subset D_{i} \subset \subset \cdots \subset \subset D_{s} \subset \subset \Omega_{s}, \\
& \Omega_{s} \subset \subset \Omega_{s_{-1}} \subset \subset \cdots \subset \subset \Omega_{j} \subset \subset \cdots \subset \subset \Omega_{1} \subset \subset \Omega_{0} .
\end{aligned}
$$


Choose $G \subset \Omega$ satisfying $D \subset \subset G \subset \subset D_{1}$. Assume $\omega \in C_{0}^{\infty}(\Omega)$ such that $\omega \equiv 1$ on $\bar{G}$ and supp $\omega \subset \subset D_{1}$. Note $I=\frac{1}{|D|} \int_{D} \omega r d x$ is average value of $\omega r$ on $D$ such that

$$
\begin{aligned}
& \left\|\omega r-\frac{1}{\left|D_{1}\right|} \int_{D_{1}} \omega r d x\right\|_{0, D_{1}} \\
= & \|\omega r-I\|_{0, D_{1}} \leq\|w\|_{1, D_{1}}+\|\omega r-I\|_{0, D_{1}} \\
\leq & \beta^{-1} \sup _{(\phi, q) \in\left(\widetilde{V}_{h}\left(D_{1}\right), M_{h}\left(D_{1}\right)\right)} \frac{B_{h}(w, \omega r-I ; \phi, q)}{\|\phi\|_{V, D_{1}}+\|q\|_{0, D_{1}}} \\
\leq & \beta^{-1} \sup _{(\phi, q) \in\left(\widetilde{V}_{h}(\Omega), M_{h}(\Omega)\right)} \frac{B_{h}(w, \omega r-I ; \phi, q)}{\|\phi\|_{V, \Omega}+\|q\|_{0, \Omega}}
\end{aligned}
$$

Apply Green Theorem [23], Theorem 2.1 and Assumptions A2, refer to Lemma 3.2 in literature [29], we have

$$
\begin{aligned}
B_{h}(w, w r-I ; \phi, q)= & a(w, \phi)-d(\phi, \omega r-I)+d(w, q)+G(\omega r-I, q) \\
\leq & c \mid w\left\|_{1, D_{1}}\right\| \phi\left\|_{1, \Omega}+c\left(\|w\|_{1, D_{1}}+\|r\|_{-1, D_{1}}+\|g\|_{-1, \Omega_{0}}\right)\right\| \phi \|_{1, \Omega} \\
& \quad+c h_{\Omega_{0}}\|\phi\|_{1, \Omega}\|r\|_{0, D_{1}}+c\|w\|_{1, D_{1}}\|q\|_{0, \Omega} \\
\leq & \|w\|_{1, D_{1}}\left(\|\phi\|_{1, \Omega}+\|q\|_{0, \Omega}\right)+c h_{\Omega_{0}}\|r\|_{0, D_{1}}\left(\|\phi\|_{1, \Omega}+\|q\|_{0, \Omega}\right) \\
& +c\left(\|w\|_{1, D_{1}}+\|r\|_{-1, D_{1}}+\|g\|_{-1, \Omega_{0}}\right)\left(\|\phi\|_{1, \Omega}+\|q\|_{0, \Omega}\right)
\end{aligned}
$$

where

$$
|G(\omega r-I, q)|=|G(\omega r, q)|=|G(r, q)|=|-d(w, q)| \leq c\|w\|_{1, D_{1}}\|q\|_{0, \Omega} .
$$

Combining with (3.3) yields

$$
\left\|\omega r-\frac{1}{\left|D_{1}\right|} \int_{D_{1}} \omega r d x\right\|_{0, D_{1}} \leq c\left(h_{\Omega_{0}}\|r\|_{0, D_{1}}+\|w\|_{1, D_{1}}+\|r\|_{-1, D_{1}}+\|g\|_{-1, \Omega_{0}}\right)
$$

then

$$
\|r\|_{0, D} \leq c\left(h_{\Omega_{0}}\|r\|_{0, D_{1}}+\|w\|_{1, D_{1}}+\|r\|_{-1, D_{1}}+\|g\|_{-1, \Omega_{0}}\right),
$$

where

$$
\left\|\frac{1}{\left|D_{1}\right|} \int_{D_{1}} r d x\right\|_{0, D_{1}}=\left\|\frac{1}{\left|D_{1}\right|} \int_{D_{1}} \omega r d x\right\|_{0, D_{1}}=\left|D_{1}\right|^{-\frac{1}{2}}\left|\int_{D_{1}} \omega r d x\right| \leq c\|r\|_{-1, D_{1}},
$$

is applied. Similarly, we have

$$
\|r\|_{0, D_{i-1}} \leq c\left(h_{\Omega_{0}}\|r\|_{0, D_{i}}+\|w\|_{1, D_{i}}+\|r\|_{-1, D_{i}}+\|g\|_{-1, \Omega_{0}}\right), \quad i=1, \cdots, s-1,
$$


where $D_{0}=D$. Thus, form Assumptions A0, A2 and the assumption on $s$, we can obtain

$$
\begin{aligned}
\|r\|_{0, D} & \leq c\left(h_{\Omega_{0}}^{s-1}\|r\|_{0, D_{s-1}}+\|w\|_{1, D_{s-1}}+\|r\|_{-1, D_{s-1}}+\|g\|_{-1, \Omega_{0}}\right) \\
& \leq c\left(\|r\|_{-1, D_{s-1}}+\|w\|_{1, D_{s-1}}+\|g\|_{-1, \Omega_{0}}\right) .
\end{aligned}
$$

Also, it is valid that

$$
\|r\|_{0, D} \leq c\left(\|r\|_{-1, \Omega_{s}}+\|w\|_{1, \Omega_{s}}+\|g\|_{-1, \Omega_{0}}\right) .
$$

The following proof is similar to $[29,30]$ and the following estimate for the stabilization term:

$$
|G(r, q)| \leq c \alpha\|r\|_{0, D_{1}}\|q\|_{0, D_{1}} \leq c h_{\Omega_{0}}^{2}\|r\|_{0, D_{1}}^{2}+c h_{\Omega_{0}}\|r\|_{0, D_{1}}^{2} \leq c h_{\Omega_{0}}\|r\|_{0, D_{1}}^{2} .
$$

We refer $[29,30]$ for details.

Theorem 3.1. Support that Assumptions A1-A3 hold. $\left(u_{H}^{h}, p_{H}^{h}\right) \in V_{H, h}(\Omega) \times M_{H, h}(\Omega)$ obtained from Algorithm 3.1 satisfies

$$
\left\|u_{h}-u_{H}^{h}\right\|_{V, D}+\left\|p_{h}-p_{H}^{h}\right\|_{0, D} \leq c H^{2}\left(\|f\|+\|g\|_{L^{2}(S)}\right),
$$

then

$$
\left\|u-u_{H}^{h}\right\|_{V, D}+\left\|p-p_{H}^{h}\right\|_{0, D} \leq c\left(h+H^{2}\right)\left(\|f\|+\|g\|_{L^{2}(S)}\right) .
$$

Proof. Setting $v=u_{h} \pm \widetilde{v}$ in (2.5) yields

$$
a\left(u_{h}, \widetilde{v}\right)-d\left(\widetilde{v}, p_{h}\right)+d\left(u_{h}, q\right)+G\left(p_{h}, q\right)=(f, \widetilde{v}), \quad \forall(\widetilde{v}, q) \in\left(\widetilde{V}_{h}, M_{h}\right),
$$

setting $v=u_{H}^{h} \pm \widetilde{v}$ in (3.1) yields

$$
a\left(u_{H}^{h}, \widetilde{v}\right)-d\left(\widetilde{v}, p_{H}^{h}\right)+d\left(u_{H}^{h}, q\right)+G\left(p_{H}^{h}, q\right)=(f, \widetilde{v}), \quad \forall(\widetilde{v}, q) \in\left(\widetilde{V}_{h, H}, M_{h, H}\right),
$$

where $\widetilde{V}_{h}, \widetilde{V}_{h, H}$ are finite element subspace of $V_{0}$. Since the assumption on the auxiliary grid $T^{h}(\Omega)$ that coincides with $T^{H, h}(\Omega)$ on $\Omega_{0}$, we can subtract the two equations, such that

$$
\begin{aligned}
& a\left(u_{h}-u_{H}^{h}, \widetilde{v}\right)-d\left(\widetilde{v}, p_{h}-p_{H}^{h}\right)+d\left(u_{h}-u_{H}^{h}, q\right) \\
& \quad+G\left(p_{h}-p_{H}^{h}, q\right) v=0, \quad \forall(\widetilde{v}, q) \in\left(V_{0}^{h}\left(\Omega_{0}\right), M_{0}^{h}\left(\Omega_{0}\right)\right) .
\end{aligned}
$$

From Lemma 3.2, we have

$$
\begin{aligned}
& \left\|u_{h}-u_{H}^{h}\right\|_{V, D}+\left\|p_{h}-p_{H}^{h}\right\|_{0, D} \\
\leq & c\left(\left\|u_{h}-u_{H}^{h}\right\|_{0, \Omega_{0}}+\left\|p_{h}-p_{H}^{h}\right\|_{-1, \Omega_{0}}\right) \\
\leq & c\left(\left\|u-u_{h}\right\|_{0, \Omega_{0}}+\left\|p-p_{h}\right\|_{-1, \Omega_{0}}+\left\|u-u_{H}^{h}\right\|_{0, \Omega_{0}}+\left\|p-p_{H}^{h}\right\|_{-1, \Omega_{0}}\right) \\
\leq & c H^{2}\left(\|f\|+\|g\|_{L^{2}(S)}\right),
\end{aligned}
$$

combine with (2.11), this theorem can be proved. 


\section{Parallel stabilized finite element algorithm}

First, assume $T^{H}(\Omega)$ to be a initial coarse grid, we divide $\Omega$ into a number of disjoint subdomain $D_{1}, D_{2}, \cdots, D_{J}$ and then enlarge each $D_{j}$ to obtain overlapping subdomains $\Omega_{j}$ such that $D_{j} \subset \subset \Omega_{j} \subset \Omega,(j=1, \cdots, J)$. For each $\Omega_{j}$, we can use some local refinement and adaptive processes to obtain a global composite mesh $T_{j}^{H, h}(\Omega)$, the composite mesh is fine inside subdomain $\Omega_{j}$ and a much coarse mesh in rest domain. All of these $T_{j}^{H, h}(\Omega)$ compose a full domain partition of $\Omega$, see Fig. 1 for a case of $J=4$. The main idea of parallel stabilization algorithm is that applying local stabilization algorithm on each global composite mesh $T_{j}^{H, h}(\Omega)$ to obtain local finite element approximate solutions. Each subproblem for $\Omega_{j}$ is defined in the entire domain with most of the degrees of freedom contained in the specified subdomain which it is responsible for, and then we can solve it in parallel with other subproblems by using an existing sequential solver without extensive recoding. For mesh $T_{j}^{H, h}(\Omega)$, the corresponding finite element space is denote as $V_{j}^{H, h}(\Omega) \subset V, M_{j}^{H, h}(\Omega) \subset M$ which satisfy the Assumptions A1-A3.
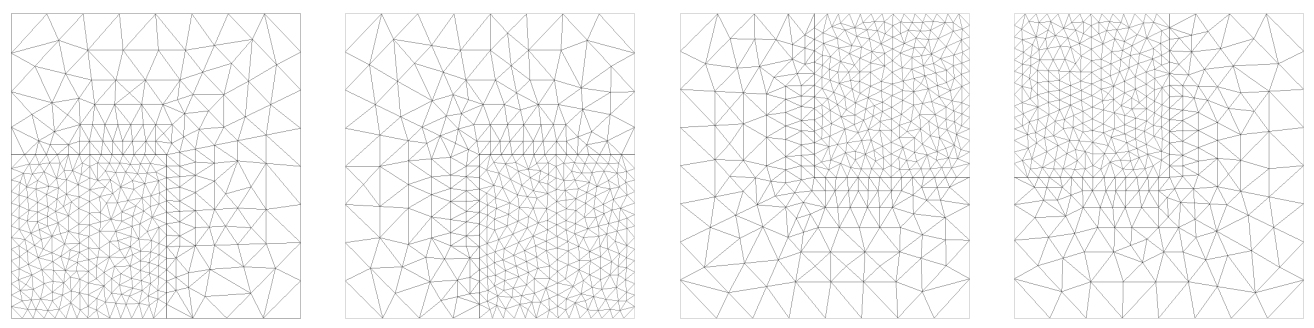

Figure 1: Full domain partition for the case of four subdomians.

Algorithm 4.1. Parallel stabilized finite element algorithm.

1. Find $\left(u_{j}^{H, h}, p_{j}^{H, h}\right) \in V_{j}^{H, h}(\Omega) \times M_{j}^{H, h}(\Omega),(j=1, \cdots, J)$ satisfies

$$
\begin{cases}a\left(u_{j}^{H, h}, v\right)-d\left(v, p_{j}^{H, h}\right)+j\left(v_{\tau}\right)-j\left(u_{\tau j}^{H, h}\right) \geq(f, v), & \forall v \in V_{j}^{H, h}(\Omega), \\ d\left(u_{j}^{H, h}, q\right)+G\left(p_{j}^{H, h}, q\right)=0, & \forall q \in M_{j}^{H, h}(\Omega) .\end{cases}
$$

2. Set $\left(u^{h}, p^{h}\right)=\left(u_{j}^{H, h}, p_{j}^{H, h}\right),(j=1, \cdots, J)$ in $D_{j}$.

Define piecewise norms

$$
\left|\left\|u-u^{h}\right\|\right|_{1, \Omega}=\left(\sum_{j=1}^{J}\left\|u-u^{h}\right\|_{1, D_{j}}^{2}\right)^{\frac{1}{2}}, \quad\left|\left\|p-p^{h}\right\|\right|_{0, \Omega}=\left(\sum_{j=1}^{J}\left\|p-p^{h}\right\|_{0, D_{j}}^{2}\right)^{\frac{1}{2}} .
$$


Theorem 4.1. Let $\left(u^{h}, p^{h}\right)$ be the finite element solution of Algorithm 4.1, satisfying

$$
\left|\left\|u_{h}-u^{h}\right\|\right|_{V, \Omega}+\left|\left\|p_{h}-p^{h}\right\|\right|_{0, \Omega} \leq c H^{2}\left(\|f\|+\|g\|_{L^{2}(S)}\right),
$$

then

$$
\left|\left\|u-u^{h}\right\|\right|_{V, \Omega}+\left|\left\|p-p^{h}\right\|\right|_{0, \Omega} \leq c\left(h+H^{2}\right)\left(\|f\|+\|g\|_{L^{2}(S)}\right) .
$$

Proof. From Theorem 3.1 yields

$$
\left\|u_{h}-u^{h}\right\|_{V, D_{j}}+\left\|p_{h}-p^{h}\right\|_{0, D_{j}} \leq c H^{2}\left(\|f\|+\|g\|_{L^{2}(S)}\right), \quad j=1, \cdots, J,
$$

by a collection of subdomains $D_{j},(j=1, \cdots, J)$, we can get the desired result (4.2) easily. Combining with (2.11), we finish the proof.

\section{Numerical experiments}

In this section, numerical experiments will be presented to verify the effectiveness and high efficiency of the parallel stabilization algorithm. The computer processor used in the numerical experiments is Intel(R) Core(TM) i3-2350M CPU $2.30 \mathrm{GHz}$, and the memory is 4GB. Referring to [31], the variational inequality (2.1) is equivalent to the following variational equation:

Find $(u, p) \in V \times M$ with and only one $\lambda \in \Lambda$, such that

$$
\begin{cases}a(u, v)-d(v, p)+\int_{S} \lambda g v_{\tau} d s=(f, v), & \forall v \in V, \\ d(u, q)=0, & \forall q \in M, \\ \lambda u_{\tau}=\left|u_{\tau}\right| & \text { a.e. in } S,\end{cases}
$$

where $\Lambda=\left\{\xi \in L^{2}(S),|\xi(x)| \leq 1\right.$, a.e. in $\left.S\right\}$.

On each composite mesh $T_{j}^{H, h}(\Omega)$, we solve equation (4.1) by Uzawa iterative methods (cf. [31]) to obtain approximate solution $\left(e_{j}, \eta_{j}\right)$. The initial value $\lambda^{0} \in \Lambda$ is given, we compute $e_{j}^{n}, \eta_{j}^{n}$ and $\lambda^{n+1},(n=0,1, \cdots)$ by

$$
\begin{cases}a\left(e_{j}^{n}, v\right)-d\left(v, \eta_{j}^{n}\right)=(f, v)-\int_{S} \lambda^{n} g v_{\tau} d s, & \forall v \in V_{j}^{H, h}(\Omega), \\ d\left(e_{j}^{n}, q\right)+G\left(\eta_{j}^{n}, q\right)=0, & \forall q \in M_{j}^{H, h}(\Omega),\end{cases}
$$

and $\lambda^{n+1}=P_{\Lambda}\left(\lambda^{n}+\rho g e_{j \tau}^{n}\right)$, in which the parameter $\rho>0, P_{\Lambda}: L^{2}(S) \rightarrow \Lambda$ satisfies

$$
P_{\Lambda}(\theta)=\sup (-1, \inf (1, \theta)), \quad \forall \theta \in L^{2}(S) .
$$


Convergence of the Uzawa iterative velocities is within a fixed tolerance of $10^{-6}$, i.e., the following condition is satisfied:

$$
\frac{\left\|u_{j}^{n+1}-u_{j}^{n}\right\|_{0, \Omega}}{\left\|u_{j}^{n+1}\right\|_{0, \Omega}}<10^{-6},
$$

where $u_{j}^{n}$ is the nth iterative solution on the composite mesh $T_{j}^{H, h}(\Omega)$.

For all $p_{h}, q_{h} \in M_{h}$, we compute stabilization term $G\left(p_{h}, q_{h}\right)$ by local Gauss integration method (cf. [19]): Define

$$
\begin{aligned}
& p_{i}^{T}=\left[p_{0}, p_{1}, \cdots, p_{N-1}\right]^{T}, \quad q_{j}=\left[q_{0}, q_{1}, \cdots, q_{N-1}\right], \\
& M_{i j}=\left(\phi_{i}, \phi_{j}\right), \quad p_{h}=\sum_{i=0}^{N-1} p_{i} \phi_{i}, \quad p_{i}=p_{h}\left(x_{i}\right), \quad i, j=0,1, \cdots, N-1,
\end{aligned}
$$

where $\phi_{i}$ is the basis function for stress with a value of 1 at point $x_{i}$ and 0 at other points. $M_{K},(K \geq 2)$ and $M_{1}$ are pressure mass matrices calculated by using $k$-order and 1 st-order Gauss integrals in each direction. $p_{i}, q_{i}$ are the value of $p_{h}, q_{h}$ at point $x_{i} . p_{i}^{T}$ is the transpose of the matrix $p_{i}$, such that

$$
G\left(p_{h}, q_{h}\right)=\alpha p_{i}^{T}\left(M_{K}-M_{1}\right) q_{j}=\alpha\left(p_{i}^{T} M_{K} q_{j}-p_{i}^{T} M_{1} q_{j}\right)
$$

\subsection{Analytical solution}

Consider $\Omega=[0,1] \times[0,1]$, the boundary is made up of $\Gamma$ and $S$ :

$$
\begin{aligned}
& \Gamma=\{(x, 0) \mid 0<x<1\} \cup\{(0, y) \mid 0<y<1\}, \\
& S=S_{1} \cup S_{2}=\{(1, y) \mid 0<y<1\} \cup\{(x, 1) \mid 0<x<1\} .
\end{aligned}
$$

Choose the appropriate $f$ such that the exact solution is given by

$$
\begin{array}{ll}
u(x, y)=\left(u_{1}(x, y), u_{2}(x, y)\right), & p(x, y)=(2 x-1)(2 y-1), \\
u_{1}(x, y)=-x^{2} y(x-1)(3 y-2), & u_{2}(x, y)=x y^{2}(y-1)(3 x-2) .
\end{array}
$$

The exact solution $u$ satisfies $u=0$ on $\Gamma, u \cdot \vec{n}=u_{1}=0, u_{2} \neq 0$ on $S_{1}$ and $u_{1} \neq 0, u \cdot \vec{n}=u_{2}=0$ on $S_{2}$. Moreover, the tangential vector $\tau$ on $S_{1}$ and $S_{2}$ are $(0,1)$ and $(-1,0)$, thus

$$
\begin{cases}\sigma_{\tau}=4 v y^{2}(y-1) & \text { on } S_{1} \\ \sigma_{\tau}=4 v x^{2}(x-1) & \text { on } S_{2}\end{cases}
$$

on the other hand, from the nonlinear boundary conditions (1.2), we have

$$
\left|\sigma_{\tau}\right| \leq g \quad \text { on } \quad S=S_{1} \cup S_{2}
$$


Table 1: Errors of approximate solution: $v=0.01, J=4, \alpha=\mathcal{O}(h)$, Extension $=h$.

\begin{tabular}{||cccccccc||}
\hline Method & $h$ & $H$ & $\mathrm{CPU}(\mathrm{s})$ & $\frac{\left\|u-u_{h}\right\|_{V, \Omega}}{\|u\|_{V, \Omega}}$ & $\frac{\left\|p-p_{h}\right\|_{0, \Omega}}{\|p\|_{0, \Omega}}$ & $R u H^{1}$ & $R p L^{2}$ \\
\hline & $1 / 16$ & $1 / 8$ & 0.078 & 0.139466 & 0.00993315 & - & - \\
Algorithm 4.1 & $1 / 36$ & $1 / 12$ & 0.374 & 0.0567627 & 0.0024423 & 1.10853 & 1.73003 \\
& $1 / 64$ & $1 / 16$ & 1.077 & 0.0293437 & 0.000840222 & 1.14675 & 1.85453 \\
& $1 / 100$ & $1 / 20$ & 2.979 & 0.0190067 & 0.0004208 & 0.973112 & 1.54947 \\
& $1 / 144$ & $1 / 24$ & 7.223 & 0.0130351 & 0.000258635 & 1.03428 & 1.33484 \\
\hline & $1 / 16$ & - & 0.14 & 0.128077 & 0.00815048 & - & - \\
Standard FEM & $1 / 36$ & - & 0.827 & 0.0537698 & 0.00228194 & 1.07028 & 1.56986 \\
& $1 / 64$ & - & 3.104 & 0.0297294 & 0.00101345 & 1.02991 & 1.4107 \\
& $1 / 100$ & - & 12.028 & 0.0180868 & 0.000552857 & 1.11353 & 1.35791 \\
& $1 / 144$ & - & 32.884 & 0.0127877 & 0.000328955 & 0.950786 & 1.4238 \\
\hline
\end{tabular}

Then the function $g$ can be chosen that $g=-\sigma_{\tau}>0$ on $S_{1}$ and $S_{2}$.

Let $v=0.01$, the iteration initial value $\lambda^{0}=1$ and the parameter $\rho=v$, stabilization parameter $\alpha=\mathcal{O}(h)$, overlapping size is $h$. We compute the finite element approximate solutions with fine meshes of size $1 / h=n^{2},(n=4,6, \cdots, 12)$ and coarse meshes of size $H$ satisfying $2 H=h^{\frac{1}{2}}$. The numerical results obtained by Algorithm 4.1 and standard finite element algorithms are presented in Table 1, the CPU time for Algorithm 4.1 are the maximum of the CPU time taken by Algorithm 4.1 over four subdomains, including the meshes generating time, the time of solving problems and the error computing time. $R u H^{1}$ and $R p L^{2}$ are the convergence rates with respect to the mesh parameter $h$ for velocity and pressure, we can obtain them by the formula $\frac{\ln \left(E_{i} / E_{i+1}\right)}{\ln \left(h_{i} / h_{i+1}\right)}$, where $E_{i}$ and $E_{i+1}$ are the relative errors corresponding to the fine mashes sizes $h_{i}$ and $h_{i+1}$, respectively. From the above tables, by comparing the parallel stabilization algorithm with standard finite element algorithm, we can find that there are no much difference in accuracy and the convergence order (see Fig. 2); However, the parallel stabilization algorithm can save a lot of computation time.

Next, the influence of the number of subdomains on approximate solutions will be investigated. Setting $h=1 / 144, H=1 / 24$ and $v=0.01$, stabilization parameter $\alpha=\mathcal{O}(h)$, overlapping size is $h$, we compute the finite element solution by Algorithm 4.1 with the number of subdomains is $2,4,8,16$. The results are listed in Table 2. Form it, we can find that the number of subdomians has little effect on the accuracy of velocity and pressure; the CPU time has significantly reduced with the increase of the number of subdomains. Combined with Tables 2, 3, we can explore the relationship between constant $c$ and $J$, overlapping degree. From Table 2, we can find the constant $c$ is lager than others when the number of subdomains is 2 and the constant $c$ gets the minimum value when the number of subdomains is 4. In Table 3, "Extension" stands for overlapping size. Generally, with the increase of overlapping size, the constant $c$ has increased slightly.

In this part, the influence of parameter $v$ on the accuracy of approximate solutions will be investigated. Setting $h=1 / 144, H=1 / 24, J=4$, stabilization parameter $\alpha=\mathcal{O}(h)$, 
Table 2: Errors of approximate solution: $v=0.01, h=1 / 144, H=1 / 24, \alpha=\mathcal{O}(h)$, Extension $=h$.

\begin{tabular}{||c|ccccc||}
\hline Method & $J$ & $\frac{\left\|u-u_{h}\right\|_{1, \Omega}+\left\|p-p_{h}\right\|_{0, \Omega}}{h}$ & $\mathrm{CPU}(\mathrm{s})$ & $\frac{\left\|u-u_{h}\right\|_{1, \Omega}}{\|u\|_{1, \Omega}}$ & $\frac{\left\|p-p_{h}\right\|_{0, \Omega}}{\|p\|_{0, \Omega}}$ \\
\hline & 2 & 1.04019 & 20.28 & 0.0211827 & 0.000427541 \\
Algorithm 4.1 & 4 & 0.639886 & 7.223 & 0.0130351 & 0.000258635 \\
& 8 & 0.717325 & 5.866 & 0.0145168 & 0.000386102 \\
& 16 & 0.692967 & 4.165 & 0.0140584 & 0.000338324 \\
\hline
\end{tabular}

Table 3: Change in constant $c: J=4, v=0.01, h=1 / 144, H=1 / 24, \alpha=\mathcal{O}(h)$.

\begin{tabular}{||c|cccccc||}
\hline Extension & $h$ & $H$ & $2 h$ & $2 H$ & $4 h$ & $4 \mathrm{H}$ \\
\hline$\frac{\left\|u-u_{h}\right\|_{1, \Omega}+\left\|p-p_{h}\right\|_{0, \Omega}}{h}$ & 0.639886 & 0.649785 & 0.645907 & 0.670006 & 0.638881 & 0.726927 \\
\hline
\end{tabular}

Table 4: Errors of approximate solution: $h=1 / 144, H=1 / 24, J=4, \alpha=\mathcal{O}(h)$, Extension $=h$.

\begin{tabular}{||c|cccc||}
\hline Method & $v$ & $\mathrm{CPU}(\mathrm{s})$ & $\frac{\left\|u-u_{h}\right\|_{1, \Omega}}{\|u\|_{1, \Omega}}$ & $\frac{\left\|p-p_{h}\right\|_{0, \Omega}}{\|p\|_{0, \Omega}}$ \\
\hline & 1 & 3.978 & 0.0128726 & 0.0169741 \\
& 0.1 & 5.788 & 0.0130029 & 0.00221808 \\
Algorithm 4.1 & 0.01 & 7.223 & 0.0130351 & 0.000258635 \\
& 0.005 & 6.895 & 0.0139456 & 0.000120794 \\
& 0.001 & 7.457 & 0.0130664 & 0.000165377 \\
\hline
\end{tabular}

overlapping size is $h$, we compute the finite element solutions by Algorithm 4.1 with $v=1,0.1,0.01,0.005,0.001$, then the results are listed in Table 4 . It can be found that, with the decrease of $v$, there has no difference in velocity error and the CPU time, but the pressure error decreases gradually.

Then, the influence of parameter $\alpha$ on the accuracy of approximate solutions will be investigated. Setting $h=1 / 144, H=1 / 24, v=0.01, J=4$ and overlapping size is $h$,
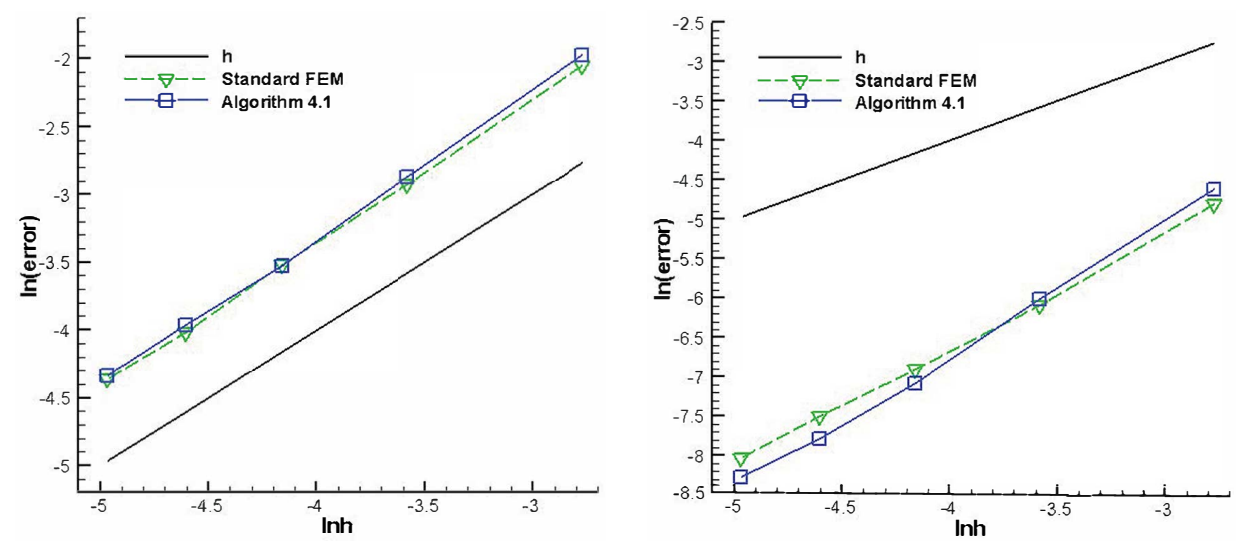

Figure 2: (a) $H^{1}$ error of the velocity, (b) $L^{2}$ error of the pressure. 
Table 5: Errors of approximate solution: $h=1 / 144, H=1 / 24, v=0.01, J=4$, Extension $=h$.

\begin{tabular}{||c|cccc||}
\hline Method & $\alpha$ & $\mathrm{CPU}(\mathrm{s})$ & $\frac{\left\|u-u_{h}\right\|_{1, \Omega}}{\|u\|_{1, \Omega}}$ & $\frac{\left\|p-p_{h}\right\|_{0, \Omega}}{\|p\|_{0, \Omega}}$ \\
\hline \multirow{5}{*}{ Algorithm 4.1 } & $h^{\frac{1}{2}}$ & 4.181 & 0.0130124 & 0.000250609 \\
& $H^{\frac{1}{2}}$ & 4.041 & 0.0130124 & 0.000250609 \\
& $h$ & 7.223 & 0.0130351 & 0.000258635 \\
& $H$ & 7.519 & 0.0130374 & 0.000259589 \\
& $h^{2}$ & 6.942 & 0.0130377 & 0.000262245 \\
& $H^{2}$ & 7.706 & 0.013037 & 0.000259345 \\
\hline
\end{tabular}

we compute by Algorithm 4.1 with $\alpha=\mathcal{O}\left(h^{\frac{1}{2}}\right), \mathcal{O}\left(H^{\frac{1}{2}}\right), \mathcal{O}(h), \mathcal{O}(H), \mathcal{O}\left(h^{2}\right), \mathcal{O}\left(H^{2}\right)$, then the results are listed in Table 5. It can be found that, the selection of the stabilization parameter $\alpha$ has little effect on the accuracy of approximate solutions.

\subsection{Numerical simulation of a bifurcated blood flow model}

In this experiment, we study a two-dimensional simplified model of blood flow in bifurcated arterial vessel. Assume that the blood vessel acts as a " $Y$ "-glyph pipe with a certain length, see Fig. 3. Blood flows into the vessel from the left entrance and out from the two outlet on the right. The inflow velocities are: $u_{1}=1.2-1.2(y-1)^{2}, u_{2}=0$. Set the diameter of the main vessel as 2 , the main branch outlet as 1.25 and another as 0.75 . The up and below boundaries of the main vessel are chosen as slip boundary with threshold function $g=\left|\sigma_{\tau}\right|$ and other boundaries are applied with Dirichlet boundary. Setting $R e=1$ and applying the Delaunay mesh generation method, 12 grid points are scattered on per unit length for the fine mesh area and 4 grid points are scattered on per unit length for the coarse grid area. Comparing standard finite element algorithm, Algorithm 4.1 with $J=2$ and $J=4$, the results are described in Figs. 4-5 respectively.

As an important geometric model in the numerical study of fluid mechanics, there are many research results for the bifurcated blood flow model (cf. [32,33]). The geometry of the blood vessels (such as bending, bifurcation, stenosis, etc.) has much affected on blood flow. In the bifurcation blood flow model, the low velocity zone will appear in the

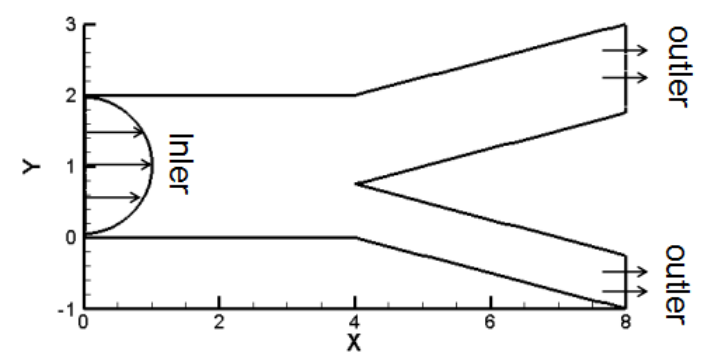

Figure 3: Computational domain of the bifurcation vascular model. 


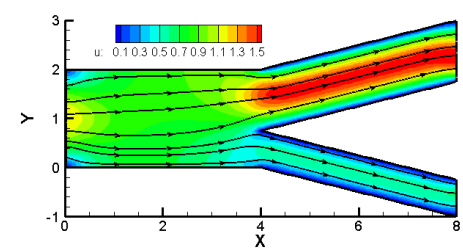

(a)

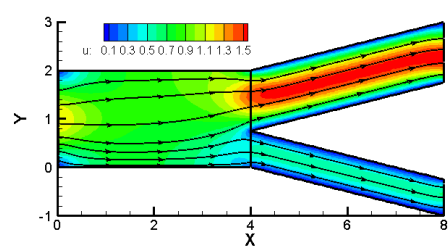

(b)

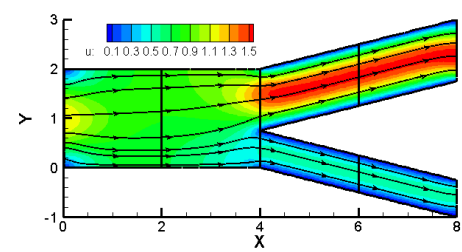

(c)

Figure 4: Velocity and streamline for standard finite element method (a), Assumption A2 with $J=2$ (b), $J=4$ (c).

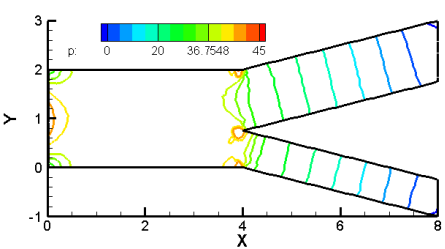

(a)

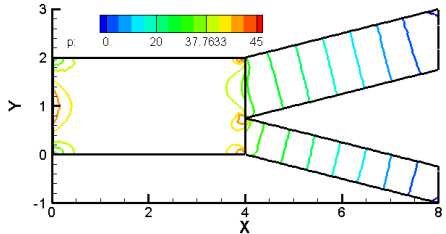

(b)

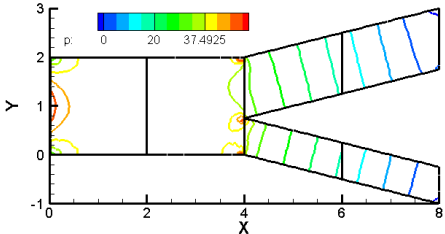

(c)

Figure 5: Pressure contours for standard finite element method (a), Assumption A2 with $J=2$ (b), $J=4$ (c).

bifurcation region where the blood flow velocity changes complexly. It causes a mutation for pressure on the vessel wall in this area. As shown in the Fig. 5, the pressure value changes slightly in the main vessel, but changes rapidly at the bifurcation junction. In this area, the contact between the blood cells and the blood vessel wall increases, the esters and mineral particles in the blood accumulate, which can easily lead to arterial stenosis.

\section{Conclusions}

The pressure projection stabilized finite element method is effective to compensate the deficiencies of the finite element pair $P_{1}-P_{1}$. Compared with the standard finite element method, numerical experiments verify the validity and efficiency of the parallel stabilized finite element method based on full domain partition. The bifurcation blood flow model further validates this conclusion.

\section{Acknowledgements}

This work was supported by the Natural Science Foundation of China (No. 11361016), the Basic and Frontier Explore Program of Chongqing Municipality, China (No. cstc2018jcyjAX0305) and Funds for the Central Universities (No. XDJK2018B032). 


\section{References}

[1] V. GiRAult AND P. A. RAVIART, Finite Element Method for Navier-Stokes Equations: Theory and Algorithms, Springer-Verlag, 1986.

[2] R. TAMAM, Navier-Stokes Equations: Theory and Numercal Analysis (3Rd edn), NorthHolland, Amsterdam, 1983.

[3] H. FuJITA, A mathematical analysis of motions of viscous incompressible fluid under leak or slip boundary conditions, RIMS Kokyuroku, 888 (1994), pp. 199-216.

[4] C. L. Roux AND A. TANI, Steady solutions of the Navier-Stokes equations with threshold slip boundary conditions, Math. Methods Appl. Sci., 30 (2007), pp. 595-624.

[5] K. TAKAHITO, On a finite element approximation of the Stokes problem under leak or slip boundary conditions of friction type, Mathematics, 2010.

[6] Y. LI AND K. T. LI, Existence of the solution to stationary Navier-Stokes equations with nonlinear slip boundary conditions, J. Math. Anal. Appl., 381 (2011), pp. 1-9.

[7] Y. LI AND K. T. LI, Global strong solutions of two-dimensional Navier-Stokes equations with nonlinear slip boundary conditions, J. Math. Anal. Appl., 393 (2012), pp. 1-13.

[8] T. KASHIWABARA, On a strong solution of the non-stationary Navier-Stokes equations under slip or leak boundary conditions of friction type, J. Differential Equations, 254 (2013), pp. 756-778.

[9] N. SAITO, On the Stokes equation with the leak and slip boundary conditions of friction type: Regularity of solutions, Publications of the Research Institute for Mathematical Sciences-Series A, 40 (2004), pp. 345-384.

[10] R. AN AND Y. LI, Two-level penalty finite element methods for Navier-Stokes equations with nonlinear slip boundary conditions, Int. J. Numer. Anal. Model., 11 (2014), pp. 608-623

[11] Y. LI AND R. AN, Penalty finite element method for Navier-Stokes equations with nonlinear slip boundary conditions, Int. J. Numer. Methods Fluids, 69 (2012), pp. 550-566.

[12] Y. LI AND K. T. LI, Penalty finite element method for Stokes problem with nonlinear slip boundary conditions, Appl. Math. Comput., 204 (2008), pp. 216-226.

[13] F. F. JING, W. M. HAN, W. J. YAN AND F. WANG, Discontinuous Galerkin methods for a stationary Navier-Stokes problem with a nonlinear slip boundary condition of friction type, J. Sci. Comput., 76 (2018), pp. 888-912.

[14] Y. N. HE, J. LI AND X. Z. YANG, Two-level penalized finite element methods for the stationary Navier-Stoke equations, Int. J. Inf. Syst. Sci., 2 (2006), pp. 131-143.

[15] J. A. LI, L. H. SHEN AND Z. X. CHEN, Convergence and stability of a stabilized finite volume method for the stationary Navier-Stokes equations, BIT Numer. Math., 50 (2010), pp. 823-842.

[16] Y. JIANG, L. Q. MEI AND H. M. WEI, A stabilized finite element method for transient NavierStokes equations based on two local Gauss integrations, Int. J. Numer. Methods Fluids, 70 (2012), pp. 713-723.

[17] Y. Q. SHANG AND J. QIN, Parallel finite element variational multiscale algorithms for incompressible flow at high Reynolds numbers, Appl. Numer. Math., 117 (2017), pp. 1-21.

[18] Y. N. HE AND J. LI, A stabilized finite element method based on local polynomial pressure projection for the stationary Navier-Stokes equations, Appl. Numer. Math., 58 (2008), pp. 1503-1514.

[19] P. Bochev, C. Dohrmann AND M. GUNZburger, Stabilization of low-order mixed finite elements for the Stokes equations, SIAM J. Numer. Anal., 44 (2006), pp. 82-101.

[20] Y. Li AND K. T. LI, Pressure projection stabilized finite element method for Stokes problem with nonlinear slip boundary conditions, J. Comput. Appl. Math., 235 (2011), pp. 3673-3682.

[21] Y. LI AND K. T. LI, Pressure projection stabilized finite element method for Navier-Stokes equations with nonlinear slip boundary conditions, Computing, 87 (2010), pp. 113-133. 
[22] J. C. XU, A Novel two-grid method for semilinear elliptic equations, SIAM J. Sci. Comput., 15 (1994), pp. 231-237.

[23] R. AdAMS AND A. Robert, Sobolev Spaces, Academic Press, New York-London, 1975.

[24] J. C. XU AND A. H. ZHOU, Local and parallel finite element algorithms based on two-grid discretizations for nonlinear problems, Adv. Comput. Math., 14 (2001), pp. 293-327.

[25] J. A. NitsChe AND A. H. SCHATZ, Interior estimates for Ritz-Galerkin methods, Math. Comput., 28 (1974), pp. 937-958.

[26] BEIRÃo DA VeIGA H, Regularity of solutions to a nonhomogeneous flows boundary value problem for general Stokes systems in $R_{+}^{n}$, Mathematische Annalen., 331 (2005), pp. 203-217.

[27] BEIRÃO DA VEIGA H, On the regularity of flows with Ladyzhenskaya shear-dependent viscous and slip or nonslip boundary conditions, Commun. Pure Appl. Math., 58 (2005), pp. 552-577.

[28] BEIRÃO DA VEIGA H, Regularity for Stokes and generalized Stokes system under nonhomogeneous slip-type boundary conditions, Adv. Differential Equations, 9 (2004), pp. 1079-1114.

[29] Y. N. HE, A. H. ZHOU AND J. LI, Local and parallel finite element algorithms for the Stokes problem, Numerische Mathematik, 109 (2008), pp. 415-434.

[30] Y. N. HE, J. C. XU AND A. H. ZHOU, Local and parallel finite element algorithms for the NavierStokes problem, J. Comput. Math., 24 (2006), pp. 227-238.

[31] Y. LI Y AND K. T. LI, Uzawa iteration method for Stokes type variational inequality of the second kind, Acta Mathematicae Applicatae Sinica, 27 (2011), pp. 303-316.

[32] A. K. Politis, G. P. Stavropoulos AND M. N. Ghristolis et Al., Numerical modeling of simulated blood flow in idealized composite arterial coronary grafts: steady state simulations, J. Biomech., 40 (2007), pp. 1125-1136.

[33] R. MANIMARAN, CFD simulation of non-newtonian fluid flow in arterial stenoses with surface irregularities, Proceedings of World Academy of Science, Engineering and Technology, 73 (2011), pp. 957. 